\title{
VEINTE AÑOS DE DISCURSO, SALUD Y ENCUENTROS TRIÁDICOS. APUNTE BIBLIOGRÁFICO
}

\author{
CARMEN VALERO GARCÉS \\ FITISPos-UAH
}

\section{RESUMEN}

Hace 20 años -1996- la llegada de población inmigrante a España comenzaba a ser ya significativa tras una década como miembro de la Unión Europea y con ello nuevos desafíos para la comunicación intercultural. Estas gentes que llegaban por tierra, mar y aire traían lenguas y culturas casi desconocidas o con poca tradición en España y que requerían de intermediarios lingüísticos y culturales para poder comunicarse con la sociedad española y a la inversa. Es el gran momento para el desarrollo en la investigación sobre la comunicación intercultural/ interlingüística. En 1997 se publica también en España el primer artículo sobre una de las muchas formas en las que se lleva a cabo la comunicación intercultural. Me refiero a la traducción e interpretación en los servicios públicos (TISP) (Valero Garcés, 1997: 267-277). Poco después se forma el grupo de investigación FITISPos (Formación e Investigación en Traducción e Interpretación en los Servicios Públicos), grupo interdisciplinar y multilingüe con dos focos de atención complementarios: investigación y formación. Estos objetivos de FITISPos, tanto formativos como de investigación, se han ido ampliando en los sucesivos proyectos y acciones desarrolladas a lo largo del siglo XXI. La investigación sobre la comunicación con la población inmigrante en el ámbito sanitario ocupa un lugar destacado. La prueba se halla en el gran volumen de publicaciones que los miembros del grupo FITISPos han sacado a la luz en estos veinte años. El principal fin de mi artículo es precisamente hacer un recorrido por aquellas publicaciones del grupo centradas en encuentros tríadicos entre personal sanitario-paciente que no conoce el español - intermediario (intérprete/mediador/traductor) que hace posible la comunicación.

PALABRAS CLAVE: servicio público de interpretación y traducción, PSIT, comunicación intercultural, comunicación con población inmigrante, comunicación en entornos sanitarios.

\section{AbSTRact}

Since 1996, and nearly a decade after becoming a member state of the European Union, Spain has experienced a substantial influx of immigrants, giving rise 
to new challenges with regard to intercultural communication. These populations arrived by land, sea, and air, bringing with them languages and cultures almost unknown or with little tradition in Spain. This resulted in a need for cultural and linguistic mediators, thereby facilitating communication between immigrants and Spanish nationals. It is the best time to conduct research on intercultural and interlingual communication. In 1997, the first article on one of the many forms in which intercultural communication is carried out was published also in Spain. I am referring to public service interpreting and translation (PSIT) (Valero Garcés, 1997: 267-277). Shortly thereafter, the research group called FITISPos (Training and Research in Public Service Interpreting and Translation) was formed. This interdisciplinary and multilingual group focuses on research and training, and their objectives have broadened upon undertaking subsequent projects and activities throughout the 21st century. One notable field of research that the FITISPos Research Group has delved into includes communication among immigrant populations in healthcare settings. The group members' contributions are further demonstrated by the numerous articles and books they have had published over the last 20 years. The overall goal of this article is to provide an overview of publications of the group centered on triad encounters with health staff, patients who cannot speak Spanish, and the intermediary (interpreter/mediator/translator) who enables communication.

KEYWORDS: public service interpreting and translation, PSIT, intercultural communication, communication with the immigrant population, communication in health environment.

Hace 20 años -1996- la llegada de población inmigrante a España comenzaba a ser ya significativa tras una década como miembro de la Unión Europea y con ello nuevos desafíos para la comunicación intercultural. Estas gentes que llegaban por tierra, mar y aire traían lenguas y culturas europeas (rumano, polaco, búlgaro), africanas (árabe, wolof) o asiáticas (chino, hindi, urdu) casi desconocidas o con poca tradición en España y que requerían de intermediarios lingüísticos y culturales para poder comunicarse con la sociedad española y a la inversa, es decir, la sociedad con los que llegan con nuevas lenguas y costumbres. Es el gran momento para el desarrollo en la investigación sobre comunicación intercultural/interlingüística.

En 1997 se publica también en España el primer artículo sobre una de las muchas formas en las que se lleva a cabo la comunicación intercultural. Me refiero a la traducción e interpretación en los servicios públicos (TISP), subárea de los Estudios de Traducción e Interpretación que inicia entonces su desarrollo en este país. El artículo en cuestión se presentó en III Encuentros de Traducción celebrados en la Universidad de Alcalá (UAH) bajo el lema de "Nuevas tendencias en los Estudios de Traducción"- y el trabajo se titulaba: "TISP ¿de qué me hablas? ¿Una nueva especialización en los Estudios de Traducción?” (Valero Garcés, 1997: 267-277). 
Poco después se forma el grupo de investigación FITISPos (Formación e Investigación en Traducción e Interpretación en los Servicios Públicos), grupo interdisciplinar y multilingüe con dos focos de atención complementarios: investigación y formación. La formación, llevada a cabo desde el año 2001, se convierte en uno de los objetivos más ambiciosos como modo de dar respuesta real a las nuevas necesidades que van surgiendo en la sociedad española y sirviéndose de los datos que la investigación le proporciona. El fin es la formación de profesionales cualificados en la comunicación con población extranjera, preparándolos con los conocimientos teóricos y las destrezas, habilidades y herramientas necesarias para actuar de enlace, no sólo lingüístico, sino también cultural, entre el personal de las instituciones y los usuarios extranjeros.

La investigación parte de tres objetivos:

- Analizar la calidad de la comunicación en los servicios públicos.

- Estudiar y contribuir al diseño de sociedades multiculturales y multilingües.

- Desarrollar e intercambiar materiales para la formación.

Estos objetivos de FITISPos, tanto formativos como de investigación, se han ido ampliando en los sucesivos proyectos y acciones desarrolladas a lo largo de este siglo xxi. La investigación sobre la comunicación con la población inmigrante en el ámbito sanitario ocupa un lugar destacado. En 2002 se organiza ya el primer congreso específico sobre TISP, para continuar desde entonces su celebración de forma periódica (2002, 2005, 2008, 2011, 2014, 2017), reflejando la propia evolución de la sociedad hacia un modelo más multilingüe.

La prueba se halla en el gran volumen de publicaciones que los miembros del grupo FITISPos han sacado a la luz en estos veinte años como resultados de investigaciones o desarrollo de materiales útiles, tanto para la población inmigrante como para los proveedores de servicios públicos.

El principal fin de mi artículo es precisamente hacer un recorrido por aquellas publicaciones del grupo FITISPos o propiciadas por sus miembros centradas en encuentros tríadicos entre personal sanitario-paciente que no conoce el español-intermediario (intérprete/mediador/traductor) que hace posible la comunicación. Se trata de investigación empírica y el método utilizado suele ser mixto e interdisciplinar, reflejo de uno de los efectos de la globalización que se manifiesta, sin duda, en un aumento de las relaciones entre las disciplinas. El resultado es una mayor interdisciplinariedad al surgir nuevas teorías o dibujar tendencias a partir de la aplicación/adaptación de presupuestos o principios de otras disciplinas como la sociología (Giddens et al., 2009), antropología (Durranti, 2009), etno- 
grafía (Dickison, 2010), la psicología (Bot, 2015) o la ética (Valero Garcés y Tipton, 2017) y no solo de disciplinas relacionadas con la lingüística aplicada o el estudio del lenguaje como son el análisis del discurso, análisis crítico del discurso, o la pragmática. Autores como Hale (2007) Vargas Urpi (2014) o Martínez Gómez (2015) o en estudios propios (Valero Garcés, 2006, 2013) insisten en ese aumento del carácter interdisciplinar de la TISP, y de cómo las disciplinas y métodos convergen y se complementan entre sí.

El corpus de trabajo de las investigaciones suele ser también mixto basándose en grupos de conversaciones grabadas in situ o de encuentros triádicos, entrevistas o datos obtenidos de encuestas y estudios de casos. El resultado es un mayor interés por el análisis del lenguaje como medio de expresión social dentro de un contexto preciso, como reflejo de unas estructuras sociales y culturales precisas cada vez más multiculturales y globalizadas.

Por orden cronológico se presentan una serie de contribuciones centradas en el análisis de la comunicación en encuentros triádicos para el periodo 1996 a 2016 desde perspectivas diferentes, pero con un objetivo común: explorar la calidad de la comunicación con la población inmigrante y su evolución en estos veinte años. El recorrido por estos trabajos nos permitirá ver cómo ha sido esa evolución y qué consecuencias ha tenido en la sociedad del siglo XXI y cómo aplicar esos resultados en la formación.

Comenzaremos por el trabajo publicado en el umbral del siglo XXI en el I Congreso Internacional sobre TISP organizados por el grupo FITISPos bajo el lema Traducción e interpretación en los servicios públicos: nuevas necesidades para nuevas realidades. En este congreso, Sánchez-Reyes Peñamaría, Durán Martínez y Martín Casado (2002) nos ofrecen, en "Argumentos a favor de la implantación de un servicio de intérpretes en los hospitales", una visión certera de la realidad. Los autores describen cómo el fenómeno de las migraciones y la movilidad poblacional llevó a plantearse la necesidad de contar con servicios de interpretación en los hospitales y centros de salud de numerosos países del mundo para enfrentarse a situaciones en las que médico y paciente no usan el mismo idioma. Su estudio describe las ventajas que el uso de intérpretes profesionales tiene sobre otras formas habituales de interpretación a las que se suele recurrir para superar las barreras lingüísticas en la práctica clínica como son la colaboración de familiares bilingües, la figura del médico con dominio de ambas lenguas y la intervención de un intérprete voluntario no cualificado. Apuntan cómo razones de índole económica y de asignación de recursos perpetúan esta situación, si bien comentan soluciones como los servicios de interpretación telefónica operativos en los Estados Unidos y el Reino Unido.

Valero Garcés (2002: 469-496), en "Interaction and conversational constrictions in the relationships between suppliers of services and immigrant 
users", ofrece un estudio de corte más lingüístico y se ocupa de los aspectos de la interacción entre los médicos y los usuarios inmigrantes cuya lengua materna no es el español en los centros de salud en España basándose en datos cuantitativos y cualitativos que provienen de encuestas y grabaciones realizadas en el norte de Madrid durante 2000-2001. El punto de partida, siguiendo a Gumperz (1982: 172-285), es el hecho de que en la actividad diaria es frecuente que se produzcan malentendidos, frustración y asincronía en la comunicación entre hablantes que comparten lengua y entorno socio-cultural, con lo cual cabe anticipar mayores problemas de comunicación cuando alguno de los hablantes no domina bien la lengua o el entorno; y estos problemas todavía son mayores cuando alguno de los participantes no sólo no domina la lengua de comunicación, sino que además actúa como puente entre las dos partes.

Profundizando en este tema, el artículo de Valero Garcés y Lázaro (2004: 522-531), titulado "Procesos de acomodación lingüística e intercambio de roles en conversaciones institucionales. El caso de las consultas entre personal sanitario y pacientes hablantes no nativos de español (PHNN)", ofrece un análisis cualitativo detallado de consultas médicas para mostrar las modificaciones lingüísticas que tienen lugar cuando se comunican en español.

Avanzando más en la descripción de la comunicación con población que no comparte la lengua de contacto, Valero Garcés y Taibi (2004: 207-228), a través de datos obtenidos en conversaciones grabadas en centros de salud entre el médico de familia y pacientes inmigrantes marroquíes en las que uno de ellos actúa como intérprete, exploran las características y dificultades de comunicación, siguiendo la línea de estudios de Drew (1997), Sarangi y Roberts (1999), Tzanne (2000) y comparan resultados con estudios previos (Valero Garcés, 2002).

Tres años más tarde, en 2005, en el II Congreso Internacional sobre TISP y bajo el tema "Traducción como mediación entre lenguas y culturas", Raga Gimeno (2005), en "Médicos, marabouts y mediadores: problemas de comunicación intercultural en la atención sanitaria a los inmigrantes de origen senegalés", presentó un trabajo centrado en aspectos más relacionados con la comunicación intercultural en las interacciones entre inmigrantes de origen senegalés y personal sanitario español. El estudio revelaba que, además de los problemas lingüísticos y administrativos, los y las inmigrantes senegaleses destacaban, por un lado, la concepción "deshumanizada" de la sanidad occidental, que no tenía en cuenta las características personales y sociales del individuo; y, por otro, la falta de tacto o de respeto con que consideraban que eran tratados. Por su parte, el personal sanitario incidía en la pasividad y escasa colaboración que solían presentar los inmigrantes de origen senegalés en las consultas médicas. Para intentar comprender el 
origen de este tipo de problemas comunicativos se analizaron las características del sistema sanitario senegalés en general, y en particular las de la sanidad tradicional, prestando especial atención al modelo de entrevista terapéutica entre paciente y marabout (curandero). Finalmente, se ofrecían diversos tipos de recomendaciones dirigidas tanto a los inmigrantes como al personal sanitario y, sobre todo, a los mediadores interculturales.

Ese mismo año, Pena (2005), en "Communication between foreign doctors and patients", publica un estudio en el que llama la atención sobre otro tema relevante en las relaciones entre profesionales y el público no especializado, como es el caso de la comunicación entre los médicos y sus pacientes en inglés. Esta comunicación se convierte en algunos casos en una ardua tarea, ya que a veces los médicos no son conscientes del uso de tecnicismos y de la falta de conocimientos de terminología médica de sus pacientes. El estudio de Pena va más allá, al centrarse en el caso de los médicos que se van a otro país a trabajar y aprender la terminología técnica, pero no pueden comunicarse con sus pacientes debido a su falta de conocimientos lingüísticos, la falta de comprensión de diferentes acentos, expresiones, etc., de la lengua de contacto. El dilema que plantea es si a la hora de formar a profesionales médicos para trabajar en el extranjero se les debe preparar para poder mantener conversaciones cotidianas o basar la formación en terminología técnica médica.

Sánchez-Reyes y Manuel Martín Casado (2005), en "Intérpretes profesionales e intérpretes ad hoc en un entorno sanitario", vuelven tras dos años de su primera publicación a analizar la realidad de la comunicación en el entorno sanitario y a distinguir entre dos categorías: intérpretes profesionales e intérpretes $a d$ hoc, denominación esta última que engloba al personal sanitario con conocimiento de la lengua extranjera pero no entrenado en destrezas interpretativas, a los familiares y amigos bilingües del paciente y a voluntarios no cualificados que se prestan a oficiar de improvisados intérpretes. Sin embargo, su estudio revela ya ciertos avances al dar ejemplos de cómo el derecho a una comunicación de calidad en la sanidad pública va abriéndose paso en las legislaciones nacionales y, simultáneamente, los facultativos van recibiendo formación para colaborar de manera efectiva con el intérprete sanitario.

En el 2005, momento de gran apogeo de la llegada de inmigrantes a España, se publica también el libro Traducción e interpretación en los servicios públicos. Contextualización, actualidad y futuro (Valero Garcés, ed., 2005), colección de artículos que recoge la situación en diferentes ámbitos. Dos artículos llaman nuestra atención. En uno de estos artículos, tal y como su título indica, "Comunicación Interlingüistica en los otros Servicios Públicos en España. Hospitales y centros de salud en la zona centro. Realidad y futuro", Valero Garcés (2005) describe la situación de la comunicación en 
los hospitales y centro de salud desde el punto de vista de aquellas personas que se dedicaban a hacer posible dicha comunicación en un momento en el que la población inmigrante suponía un porcentaje cada vez mayor. El otro artículo, de Barroso Pérez (2005), "Problemas de comunicación en la atención a inmigrantes en los servicios sanitarios", habla directamente de esos problemas de comunicación, pero desde la perspectiva del personal sanitario de la mano de una profesional del Hospital Ramón y Cajal de Madrid, uno de los centros de referencia en la atención a la población inmigrante con una unidad de atención sobre enfermedades tropicales.

Ese mismo año se publica también un artículo en una revista de referencia internacional en el campo de la interpretación, Interpreting (Valero Garcés, 2005. "Doctor-Patient Consultations in Dyadic and Triadic Exchanges"), en el que se analizan interacciones de dos tipos: médicos y pacientes extranjeros que no conoce el español y médicos-pacientes extranjeros e intérpretes que hacen de puente de comunicación. Ello sirve para analizar las diferencias entre esos dos tipos de encuentros, habituales en esos momentos en el sistema español donde no había -ni hay- intérpretes profesionales, sino intérpretes ad hoc, que son con frecuencia familiares, amigos o voluntarios.

En otro estudio publicado en 2007 bajo el título "Modes of communication between suppliers of services and non-native English-speaking users: doctor-patient interaction" (Valero Garcés y Downing, 2007), utilizando un corpus de conversiones médicas grabadas en este caso en Estados Unidos, se lleva a cabo el análisis del discurso de las mismas para ilustrar los problemas no solo lingüísticos sino también culturales y específicos de la especialización y las ventajas de contar con intérpretes profesionales para evitar riesgos y malentendidos. Se insiste en la necesidad de formación de estos expertos de la comunicación que deben de conocer no solo el lenguaje especializado sino también el lenguaje coloquial, dadas las grandes diferencias (educativas, económicas, sociales) que pueden existir entre los profesionales y sus pacientes.

En el 2008 se celebra el III congreso de la TISP bajo un tema específico: TISP: Investigación y Práctica en Traducción e Interpretación en los Servicios Públicos. Desafios y alianzas. El objetivo, al igual que los congresos de 2002 y 2005, fue continuar con esa labor e insistir en el contacto entre la comunidad profesional y académica, las autoridades educativas competentes y las instituciones públicas y privadas, así como con futuros traductores e intérpretes, nuevos investigadores y todo el público interesado en la comunicación intercultural en general y en la traducción e interpretación en particular como herramienta necesaria en la configuración de la nueva realidad social que se está formando a nivel mundial. Año tras año se había ido avanzando y tejiendo una red cada vez más tupida. En 2008 el tema 
central fue la búsqueda de alianzas entre la investigación y la práctica de la traducción e interpretación en los servicios públicos. Ello implica riesgos, desafíos y búsqueda de alianzas entre todos los factores y agentes que intervenían (o podían intervenir): intérpretes y traductores profesionales, formadores, investigadores, educadores, voluntarios bilingües que hacen de intermediarios lingüísticos, funcionarios, profesionales de otros sectores (abogados, médicos, educadores), ONGs que realizan labores de mediación interlingüística, o representantes de instituciones o servicios públicos que se enfrentan diariamente a problemas de comunicación, políticas nacionales e internacionales, relaciones de poder, cuestiones de género, reconocimiento de las minorías culturales, etc.

En este congreso se presentan los resultados del proyecto I $+\mathrm{D}$ coordinado (REF HUM2004-03774-CO2-02/FILO) sobre comunicación interlingüística e intercultural, llevado a cabo por la Universidad de Alcalá y la Universidad Jaume I en el periodo 2004-2007. El Grupo FITISPos llevaba ya una década trabajando en el campo de la comunicación interlingüística e intercultural y había llevado a cabo varios proyectos de investigación centrados en el tema de la comunicación en los servicios públicos (hospitales, ayuntamientos, comisarías, etc.) con población extranjera. Fruto de estos análisis fueron varias propuestas y el desarrollo de materiales didácticos y de sensibilización dirigidos a los profesionales sanitarios.

En 2008 se presentan algunos de los resultados del proyecto I+D derivados de encuestas y de la transcripción de las grabaciones, contando con la colaboración de personal que dominaba las distintas lenguas reflejadas en ellas (árabe, polaco, rumano, ruso, inglés, búlgaro, francés). Su análisis revela una vez más, pero con un volumen de datos mayor -y tras una década de contactos- la defectuosa calidad de la comunicación con la población extranjera en el ámbito sanitario. Las conclusiones son las siguientes:

1. En relación con la atención a pacientes cuya lengua materna no es el español, 72 proveedores de servicios (53,33\%) de un total de 127 contestaron que alguna vez recibían pacientes de estas características; $61(45,18 \%)$ contestaron que muchos de sus pacientes no tenían el español como lengua materna, mientras sólo 2 declararon que apenas acudían a su centro pacientes con esas características.

2. En relación con los problemas lingüísticos que los pacientes inmigrantes suelen tener, 118 profesionales sanitarios $(92,04 \%$ de un total de 127 respuestas) responden que dichos pacientes tenían problemas en comprender al personal sanitario; $104(81,87 \%)$ en responder; $86(67,71 \%)$ en leer informes escritos; $80(62,98 \%)$ en leer los folletos informativos; $77(60,67 \%)$ en entender formularios para rellenar y $75(59,90 \%)$ en rellenar formularios. Finalmente 115 
profesionales $(90,55 \%)$ creían que los inmigrantes tenían especiales problemas con el lenguaje escrito (historial, volantes e informes de alta, consentimientos informados), mientras que sólo 12 (9,38\%) creían que no.

3. En cuanto a los usuarios, cuando se les preguntó cuáles eran los aspectos en los que tenían problemas, todos afirmaban que tanto en la expresión como en la comprensión oral y escrita; 27 (47,32\%) de un total de 57 dijeron que no eran capaces de comprender al personal sanitario; $29(50,82 \%)$ dijeron que se sentían inseguros a la hora de responder, otros tantos (27 o 47,32\%) sostenían que tenían dificultades en leer folletos informativos e informes escritos, y muchos encontraron obstáculos a la hora de entender formularios $(31$ o $54,39 \%$ ) y de rellenarlos (32 o $56,14 \%$ ).

4. En relación con las soluciones que buscaron para resolver los problemas lingüísticos indicaron que: a veces recurrían a familiares y amigos que conocían ambos idiomas, y que a veces se hacía necesaria la presencia de intérpretes profesionales y/o voluntarios de ONGs o de las propias embajadas. En el caso de que nadie les acompañase o no pudiesen disponer de un servicio de interpretación, los usuarios afirmaron que se servían de otras soluciones como utilizar otras posibles lenguas comunes con el personal sanitario, ayudarse de diccionarios y de folletos traducidos o incluso explicarse por señas y gestos. Muchos afirmaron que estaban intentando aprender la lengua española. Una minoría afirmó que no se resuelven nunca satisfactoriamente.

5. Cuando se les preguntó sobre recursos escritos en su lengua, la mayor parte (43 de 48, casi un 90\%) de los usuarios que respondió a esta pregunta indicaron que hay muy pocos documentos traducidos a diferentes lenguas y a la pregunta de si tienen problemas con el lenguaje escrito en español (recetas, historiales, volantes), 40 de los 55 usuarios que respondieron $(72,72 \%)$ contestaron afirmativamente.

El análisis de las transcripciones de las grabaciones de consultas reveló que la asimetría característica de los encuentros médico-paciente aumentaba cuando éste último no compartía la lengua materna del médico. Esto provocaba que, con frecuencia, no se estableciese el clima de confianza necesario entre ambos para que, en primer lugar, el profesional sanitario obtuviese suficiente información de su paciente para realizar un diagnóstico adecuado, y, en segundo lugar, que el paciente no siguiese correctamente las instrucciones dadas por el médico en cuanto a tratamiento y hábitos de vida. 
Con estos resultados se pasó a la acción que se materializó, de acuerdo con la filosofía del grupo FITISPos, en dos hechos:

1. La incorporación de materiales en las clases: uso de consultas reales grabadas utilizadas para la discusión en clase; visualizar y comentar conversaciones con profesionales de la medicina; dar a conocer las dificultades de los propios alumnos o colaboradores para conseguir el material, etc.

2. La elaboración de guías multilingües en distintos ámbitos para minimizar la problemática comunicativa. Se determinó, en primer lugar, el tema y número de las mismas dependiendo de las necesidades más urgentes encontradas y de nuestra capacidad para producirlas -sobre todo económica-. Seis fueron los temas elegidos:

- Atención a menores (pediatría).

- Atención a mujeres embarazadas y en el parto.

- Atención al inmigrante en los servicios sociales.

- Prevención de riesgos laborales (empleadas de hogar).

- Prevención de riesgos laborales (construcción).

- Primeros pasos de atención al inmigrante y refugiado (comisarías, aduanas, gobierno civil...).

Los idiomas elegidos, de acuerdo con el porcentaje de población extranjera en Madrid y la zona del Corredor del Henares fueron -por orden alfabético- árabe, búlgaro, francés, inglés, polaco, rumano y ruso. Determinados los temas y las lenguas de trabajo, se pasó a la creación de un documento base en español. Para ello, se consultaron documentos similares y se mantuvieron entrevistas con especialistas médicos y funcionarios de los servicios sociales de los ayuntamientos, sindicatos y ONGs, así como de los propios usuarios y algunos proveedores de servicios. El resultado fueron las siguientes guías multilingües:

- Guía básica multilingüe de atención al paciente, basada en otra guía anterior, pero a la que se incorporaron otros idiomas (chino y polaco).

- Guía multilingüe de atención a mujeres embarazadas.

- Guía multilingüe sobre pediatría.

- Guía multilingüe de atención al inmigrante en los servicios sociales.

- Guía multilingüe de hábitos saludables. Atención al menor (de 0 a 18 años).

Dichos materiales fueron reeditados varias veces en varios formatos y se distribuyeron de forma gratuita a todos los hospitales y centro de salud del SESCAM de la Junta de Comunidades de Castilla-La Mancha.

Otros trabajos que se publicaron en 2008 fueron los siguientes: 
Pena y Porto (2008), en “Interpreting doctors and patients' mental images of illness in community healthcare", ofrece una nueva vía de estudio del discurso al centrarse en el uso de metáforas utilizando la lingüística cognitiva como marco teórico. Partiendo de la hipótesis de que las metáforas están en la base de nuestro sistema cognitivo y es por medio de proyecciones metafóricas como comprendemos la mayoría de los conceptos abstractos, se centran en el campo de la salud, para mostrar cómo en la traducción del inglés al español una traducción descuidada de dichas metáforas puede conducir a una pérdida de metáforas conceptuales y entonces los pacientes no pueden construir la imagen mental adecuada con obvias consecuencias para su salud.

La necesidad de ofrecer formación se va convirtiendo poco a poco en un tema recurrente en la literatura científica y en el ámbito de la salud; sobre todo se plantea la discusión sobre qué formación debería recibir el intermediario para atender a las necesidades que han surgido. El debate se centra entre dos figuras: el intérprete o el mediador. Dalila (2008), en su trabajo "Un estudio de campo realizado en tres hospitales de la provincia de Alicante: impresiones sobre el papel del intérprete”, muestra ese debate para insistir en que en un momento en el que en España casi todo estaba por hacer en lo que a interpretación en los servicios públicos se refiere, desde el mundo académico debería tomarse la iniciativa de la formación y, junto con el ámbito profesional, la elaboración de códigos deontológicos y normas de buenas prácticas que ayudasen a definir el papel del intérprete, o mediador interlingüístico e intercultural. Tal debate sigue abierto hoy en día.

Prieto (2008), centrándose en la interpretación telefónica, insiste también en la formación a través de una experiencia llevada a cabo en un hospital de Málaga y apoyándose en la figura del mediador intercultural e interlingüístico más que en la del intérprete como persona que trasmite fielmente lo que escucha para proponer soluciones.

La idea de que el discurso en el ámbito sanitario es diferente al de otros ámbitos -por ejemplo el jurídico- en el caso de la actuación de los intérpretes en encuentros triádicos va quedando cada vez más patente en la investigación, como lo demuestra el trabajo de Rodríguez Navaza (2008), "África, sangre y VIH: el papel del mediador en la resolución de conflictos culturales específicos en el ámbito sanitario”. Su investigación pretende profundizar en las representaciones culturales de la sangre en pacientes de origen subsahariano con el fin de detectar aquellos factores que hacían que muchos de ellos se mostrasen reacios a hacerse análisis de sangre en España. A partir de los resultados, se debate el papel del mediador intercultural para superar esta barrera específica que en muchos casos supone el alejamiento de estos inmigrantes del sistema de salud español y la de- 
tección tardía de enfermedades. Como conclusión se presentan estrategias específicas de mediación intercultural aplicadas en programas de prevención y promoción de la salud dirigidos a este colectivo.

Fuera del congreso organizado por el grupo FITISPos en 2008, salen también a la luz otras publicaciones centradas en la TISP en el ámbito sanitario mostrando el interés por esta área en un momento en el que en España todavía no había estallado la crisis y se estaban articulando algunas soluciones que quedaron más tarde olvidadas. Podemos citar el capítulo titulado "Salud, Comunicación e Inmigración" (Valero Garcés, 2008: 381-402), publicado en el Manual sobre Comunicación e Inmigración editado por Bañón y Fornieles. En este artículo se lleva a cabo el análisis de los elementos que intervienen en la cadena de comunicación lingüística, los diferentes tipos de encuentros que se producen en las consultas médicas y los problemas que se plantean. Se dan también a conocer los resultados de algunas investigaciones, aportando ejemplos reales de conversaciones en consultas médicas para finalmente aportar algunas soluciones de cara al futuro, con especial énfasis en la figura del intermediario o tercer eslabón de esa cadena de comunicación que hace posible el salvar las dificultades que el idioma y la cultura plantean.

Otros ejemplos de análisis del discurso basado en conversaciones reales y desde diferentes perspectivas son los trabajos publicados en foros internacionales como es el caso del capítulo "Hospital interpreting practice in the classroom and the workplace" (Valero Garcés, 2010) y "Challenging communication in Doctor/Non-Native Patient Encounters. Two perspectives, three types of interaction and some proposals" (Valero Garcés y Downing, 2010). En 2010 Lázaro Gutiérrez defiende la Tesis Doctoral "Análisis del discurso institucional entre hablante nativo (HN) y hablante no nativo (HNN) en los servicios públicos", la primera tesis publicada en nuestro país centrada en el ámbito sanitario.

FITISPos vuelve a organizar el IV Congreso sobre TISP en 2011. El objetivo principal es continuar con esa misma labor de diálogo e intercambio de experiencias y proyectos de ediciones anteriores. El tema en esta ocasión, acorde con los tiempos que corrían, fue El futuro en el presente: Traducción e interpretación en los Servicios Públicos en un mundo INTERcoNEcTado. Son varios los trabajos que se publican referidos al ámbito sanitario. Algunos son estudios de caso como el de Clavero Curado (2011) con "La documentación sanitaria para personas no hispanohablantes en Andalucía. Análisis y propuestas", donde ya van llegando ecos de la crisis al poner de manifiesto la necesidad de tener en consideración las limitaciones de los recursos y plantear herramientas para prevenir situaciones de desigualdad lingüística. 
Vitalaru (2011), en "Herramientas wiki, trabajo colaborativo y elaboración de materiales multilingües de carácter sanitario en un máster universitario", da cuenta de dos actividades desarrolladas y aplicadas, mediante las herramientas wiki, en el Máster Universitario Europeo en Comunicación Intercultural, Interpretación y Traducción en los Servicios Públicos en los cursos académicos 2009-2010.

Faya Ornia (2011), por su parte, en “Trabajo de campo: tecnologías empleadas en los servicios de interpretación de los hospitales (Londres, Düsseldorf y Madrid) y experiencias de los usuarios", presenta una experiencia similar. Valenciano Rodríguez y Bueno Guillén (2011) recogen un estudio comparativo entre el uso de vídeos en lengua de signos y avatares signantes en el ámbito sanitario, mediante entrevistas a personas sordas usuarias de la lengua de signos española.

En 2011 se publica también el trabajo "La conversación de contacto en contextos institucionales: la consulta médica" (Valero Garcés, 2011), donde se analiza un aspecto poco estudiado dentro de la prolífica línea de análisis del discurso institucional como es el análisis de la conversación de contacto en un contexto específico -la consulta médica-. Los resultados de este estudio demuestran que ambos tipos de comunicación son inseparables y centrales y aparecen entrelazados en las distintas partes de la entrevista. Asimismo, el tono coloquial de las mismas y la presencia de usuarios extranjeros que no domina bien la lengua y/o el funcionamiento del sistema sanitario español favorece su uso.

$\mathrm{Al}$ año siguiente, en 2012, FITISPos organiza el primer congreso internacional sobre comunicación en el ámbito sanitario (1st International Symposium on Health Care Interpreting Communicating in the Healthcare Setting (Valero Garcés, ed., 2012). En la publicación derivada del mismo los distintos autores abordan aspectos varios y se pone de manifiesto que, a pesar de haberse arbitrado algunos mecanismos para satisfacer las necesidades comunicativas, las iniciativas existentes todavía reflejan una falta de estructuras profesionales y de interés por la calidad. Esta publicación resulta especialmente pertinente en el momento que vivía la profesión y la disciplina de la TISP en España, atenazada ya por la crisis con la supresión de las pocas ayudas institucionales para investigación y/o formación y el cierre de algunos servicios o infraestructuras que se habían iniciado en años anteriores (por ejemplo, el servicio de mediación del ayuntamiento de Madrid). En este contexto, la aportación de la asociación internacional de intérpretes médicos (Internacional Medical Interpretes Association (IMIA)) supone un apoyo importante por su experiencia para motivar la profesionalización de la actividad y la defensa de los derechos de los usuarios. Los temas tratados dan cuenta de este interés: 
- Avances en la investigación y práctica de la TISP en el ámbito médico.

- Relaciones de empresas y práctica de la TISP en el ámbito médico.

- Las instituciones y sus relaciones con las entidades formadoras.

- La interpretación médica y los códigos deontológicos.

Un año más tarde, en 2013, en Panace@ se incluye el artículo "Iniciación a una base de datos sobre artículos de investigación" (Carmen Valero Garcés, 2013). El objetivo era doble. Por un lado, se buscaba contribuir a llenar ese hueco entre formación y práctica y, por otro lado, pretendía profundizar en las relaciones entre dos áreas bien definidas: humanidades y ciencias médicas, y más específicamente, entre los estudios sobre comunicación intercultural y la atención sanitaria a población extranjera. El modo para cumplir dicho objetivo fue analizar estudios llevados a cabo por futuros especialistas de la comunicación en el marco del Master Universitario Europeo en Comunicación Intercultural, Interpretación y Traducción en los Servicios Públicos. Algunos de los temas explorados en sus trabajos fueron la influencia de traductores e intérpretes en la educación sexual en un análisis comparativo de China y España; el análisis de los límites del papel del intérprete en los hospitales de Madrid y Bruselas; el impacto psicológico y emocional en los intérpretes en el análisis de casos y situaciones en Turquía y España; la importancia del origen cultural del intérprete o mediador en los servicios públicos en el caso del chino-español; o el análisis de diferencias culturales que puedan afectar al vocabulario y en consecuencia, las interpretaciones o mediaciones. La variedad de temas y de lenguas y culturas que se manejan dan idea de la importancia de estas investigaciones para profundizar en el conocimiento de nacientes sociedades multiculturales. Los trabajos se agruparon en las siguientes categorías:

- Comunicación intercultural y percepciones de la población extranjera y/o de las instituciones sobre aspectos culturales.

- La salud y la atención sanitaria a población extranjera.

- El papel(es) de los intermediarios o personas que sirven de enlace en las consultas médicas o visitas a la administración y su profesionalización.

- Disponibilidad y calidad de materiales multilingües de apoyo.

En 2014, FITISPos organiza el V congreso sobre TISP bajo el tema: $(R e)$ Considerando ética e ideología en situaciones de conflicto en la TISP, con el fin de mostrar otros discursos que empezaban a hacerse visibles. Comenzamos el siglo invadidos por el término "globalización", pero trascurrida la primera década y superadas las grandes expectativas y miedos que el inicio de un 
nuevo siglo presagiaba se descubren otros efectos, no tan positivos. Estos efectos se percibían también en la TISP. Inmersos en este ámbito como investigadores, formadores y practicantes de la TISP desde hace casi dos décadas (Valero Garcés, 1997), hemos ido observando el nacimiento y su evolución en España al compás de los tiempos, tal y como dan fe las publicaciones antes mencionadas.

Con la crisis, la inmigración ha cesado en algunas partes del planeta y la atención se va derivando hacia otros problemas que van calando poco a poco en las conciencias de los ciudadanos y ampliando las áreas en las que los intérpretes y traductores deben intervenir: violencia de género, conflictos laborales, desarraigo escolar, violaciones de los derechos humanos o extradiciones. Y en el plano internacional, los conflictos armados abiertos, los campos de refugiados, los juicios por crímenes de guerra o la violación de los derechos humanos provocan similares efectos y dibujan la necesidad de observar diferentes principios éticos para diferentes situaciones. Todo ello influye, sin duda, en la labor del intérprete y traductor y plantea cuestiones éticas e ideológicas. En 2014 había ya investigaciones y publicaciones de diferente signo que daban sobrada cuenta de este hecho (Hale, 2005: 15; Inghilleri, 2005; Stahuljak, 2000; o Mona Baker, 2006) que analiza discursos y relatos de traductores en situaciones de conflicto para plantear cuestiones éticas y modelos de estudio de dichas narrativas. Surgen también estudios sobre los principios éticos que deben regir la actuación de estos traductores e intérpretes y que demuestran conflictos no solo internos, sino también externos. Los trabajos en Estados Unidos de Kearns (2006) o Kenigson-Kristy (2005) y Lafranchi (2006), relacionados con el impacto del ataque a las Torres Gemelas y sus consecuencias, o los trabajos de Martin y Taibi (2012) o Valero Garcés y Abkari (2011), relacionados con el atentado terrorista en la red de trenes de cercanías de Madrid el 11 de marzo de 2004, son algunos ejemplos.

Todo lo dicho en los párrafos anteriores son ejemplos que, sin duda, plantean cuestiones éticas e ideológicas. Tanto en el ámbito de la investigación como en la información cada vez hallamos más estudios o relatos de casos. Cabe no obstante comentar la paradoja de esta realidad: cada vez hay más material sobre aspectos relacionados con la actividad de los traductores e intérpretes en situaciones de conflicto, pero es más cualitativo que cuantitativo y, por lo tanto, más subjetivo que objetivo, pero la consideración del discurso como objeto de análisis e hilo conductor en muchas de estas investigaciones es una realidad.

Algunos artículos incluidos en la publicación derivada del V Congreso con el mismo título de (Re) Considerando ética e ideología en situaciones de conflicto en la TISP (2014) dan fe de ello. Investigaciones sobre cuestiones éticas derivadas de la actuación de intérpretes y traductores en situacio- 
nes de conflictos armados; descripciones de acciones llevadas a cabo por intérpretes y traductores que han intervenido en casos de víctimas de guerra, abuso sexual o solicitudes de asilo y refugio; debates sobre conflictos y enfrentamientos ideológicos que surgen en el campo de traducción e interpretación; o análisis de aspectos relacionados con los avances de TISP en las instituciones locales, nacionales e internacionales, bajo la influencia en la Unión Europea de la Directiva 2010/64/UE del Parlamento Europeo y del Consejo de 20 de octubre de 2010 relativa al derecho a interpretación y a traducción en los procesos penales, son temas cuyos discursos muestran diferencias y similitudes.

Si nos centramos en el ámbito sanitario, merece la pena mencionar los trabajos derivados del proyecto $\mathrm{I}+\mathrm{D}+\mathrm{i}$ titulado "InterMed: Mediación interlingüística e intercultural: Diseño, coordinación y seguimiento de mediadores sanitarios" (Ref FFI2011-25500). Gracias al proyecto se implantó un servicio de traducción e interpretación en los centros de salud de Parla, un municipio al sur de Madrid, formado por tres mediadoras que prestan servicios lingüísticos a profesionales sanitarios y pacientes en chino, francés, inglés y rumano. Las mediadoras evaluaron las necesidades en materia de comunicación interlingüística e intercultural mediante la realización de encuestas a estos dos grupos y llevaron a cabo un seguimiento de la organización del equipo de mediadoras a través de un registro de las intervenciones y de informes semanales del trabajo realizado en cada idioma. A dicho proyecto pertenecen los artículos de Jaime (2014), con "Acogida del Servicio de Interpretación en Centros de Salud de Parla: primeras impresiones", que describe el proyecto; Jiménez Hortelano (2014) con "Mediación e Interpretación de Chino en el proyecto InterMed", que presenta los resultados obtenidos de las actuaciones con población china. Por su parte, Ilie (2014), con "Mediación e interpretación de inglés y rumano en el proyecto InterMed", que presenta -como el título indica- los resultados del proyecto piloto en el caso de inglés y rumano.

Ese mismo año, 2014, Panace@, dedica un monográfico a la Comunicación intercultural en el ámbito médico. Los artículos incluidos ayudan a desentrañar algunos interrogantes que surgen en la convivencia de lenguas y culturas tras unos primeros encuentros, explorando sinergias clave y discordancias entre el mundo profesional y la vida real de la interpretación médica. A lo largo de los capítulos se recogen debates teóricos y estudios de caso como muestra de la amplia temática que ofrece la comunicación intercultural y de lo mucho que queda aún por hacer. Entre otros temas, se reflejan aproximaciones diferentes al concepto de comunicación intercultural (Nevado Llopis y Sánchez-Pedreño Sánchez); investigaciones sobre los retos y dilemas que supone para los intérpretes médicos el "ser éticos" y "actuar de manera ética" (Baixauli); estudios sobre las implica- 
ciones de la (in)visibilidad del intérprete médico en situaciones específicas (Ilie, Jaime Pérez, Vitalaru); o el análisis de la influencia de las perspectivas del personal sanitario y de los pacientes en la interpretación médica (Valero Garcés y Wahl); o incluso el análisis de materiales multilingües en España (Marnpae).

Al año siguiente, 2015, se publica una monografía dedicada al ámbito sanitario en inglés, contribuyendo así a dar proyección internacional a la situación en España. Se trata de la obra Health, Communication and Multicultural Communities. Topics on Intercultural Communication for Healthcare Professionals. Se trata más de un manual dirigido a los proveedores de servicios y a los profesionales de la salud, y se profundiza en conceptos con los que están familiarizados los expertos del lenguaje (es decir, los modos de interpretar, el valor de la comunicación intercultural, la importancia de la profesionalización, etc.). Es, además, un libro de cierto tono pedagógico como herramienta valiosa para los formadores de los futuros médicos e intérpretes médicos. Algunos temas tratados son, por ejemplo, el estudio de las distintas percepciones sobre el uso de la lengua de los participantes en encuentros tríadicos; el uso del inglés como lengua franca; la negociación de significado en las consultas médicas; el uso de estrategias de gestión en las relaciones interculturales en encuentros médicos o el trabajo en equipo de los profesionales de la salud y de la comunicación.

Este interés por el ámbito sanitario lleva al grupo FITISPos a participar también en el proyecto europeo Speak Out for Support (SOS-VICS) (JUST/ 2011/JPEN/AG/2912), un proyecto piloto, cofinanciado por el Programa Justicia Penal de la Unión Europea y las universidades socias, cuyo objetivo es mejorar la formación de intérpretes en el ámbito de la violencia de género (VG) y el resultado fue la publicación, entre otros materiales, del artículo "Interpretar en casos de violencia de género en el ámbito médico" (Valero Garcés y Lázaro, 2015: 192-238).

En el 2016 aparece una nueva publicación en las que se incluyen artículos relacionados con el ámbito sanitario. Me refiero a Public Service Interpreting and Translation (PSIT): training, testing and accreditation / Traducción e Interpretación en los Servicios Públicos (TISP): formación, evaluación y acreditación. En dicha publicación se incluyen los trabajos de Jiménez y Murgu centrados en la interpretación telefónica, forma de comunicación que goza cada vez de mayor aceptación. Se analizan partes de discurso auténtico y se proponen ejercicios. Nevado Llopis dibuja el mapa de las competencias que un intérprete profesional debería poseer hoy en día, y Pérez Estevan se centra en el tema de poder entre el intérprete y el profesional de la salud.

En 2016 se lee la tesis "La traducción de textos (inglés-español) en el ámbito de la salud mental: rasgos e implicaciones a través de un estudio contrastivo", de Echauri Galán, en la que se trata un tema cada vez más 
común en las relaciones interculturales en este siglo xxi: la salud mental. Mientras, siguen en marcha otros proyectos y tesis doctorales que pronto verán la luz como, por ejemplo, el análisis de aspectos lingüísticos y traductológicos de la traducción médica en el caso del español-árabe de Mohana Sultan. La lista podría ser más larga, pero creemos que hay ya material suficiente para reflexionar sobre la importancia de la investigación sobre la salud y la comunicación intercultural en nuestro entorno.

\section{ConClusiones}

El breve recorrido por las publicaciones referidas a la salud en este siglo muestra esa evolución de las relaciones en encuentros tripartitos yendo desde descripciones de la situación entre hablantes de lenguas y culturas distantes hasta cuestiones ya derivadas de un conocimiento de años como son cuestiones éticas, de consolidación de una nueva profesión o relacionado con la salud mental. Y en todo ello el discurso es pieza fundamental en la investigación.

El marco teórico sobre el que se apoyan las aportaciones que hemos mencionado se basa, como se ha indicado ya, en el análisis de encuentros triádicos en los que uno de los elementos hace de enlace asumiendo el papel de intermediario (intérprete/mediador/traductor) en un contexto institucional específico (entrevista médico-paciente). Las investigaciones revelan datos interesantes en cuanto a la forma y calidad de la comunicación desde perspectivas distintas (análisis del discurso, comunicación intercultural, análisis del discurso institucional) y se observaban cambios importantes al modificarse la estructura estándar de este tipo de interacciones institucionales cambiando la rutina que las hace más funcionales. Se economizan esfuerzos y se ajustan a las limitaciones espacio-temporales a las que esas interacciones se ven restringidas. Además, el hecho de que haya un tercer elemento cuya función es servir de puente entre el médico y el paciente añade otras características a la interacción y revela igualmente nuevas deficiencias en la comunicación, como son la falta de preparación del intérprete para desarrollar dicho papel, la transmisión defectuosa de la información y la necesidad de una negociación intensa por las partes para lograr la comunicación, lo cual equivale a más tiempo y esfuerzo con los consiguientes efectos en el sistema sanitario español.

En definitiva, este análisis no hace sino constatar una situación todavía cotidiana en el sistema sanitario español, así como cierta evolución en la temática que recoge las investigaciones en el periodo 1996-2016. 


\section{BIBLIOGRAFÍA}

BaiXauli Olmos, L. (2014): "Redescubriendo los códigos de conducta para la ISP médica: cultura, dilemas y papel profesional", Panace@, 5,1.

BAKer, M. (2006): Translation and Conflict: A Narrative Account, Londres/Nueva York, Routledge.

Barroso Pérez, E. (2005): "Problemas de comunicación en la atención a inmigrantes en los servicios sanitarios", en C. Valero Garcés (ed.), Traducción e interpretación en los servicios públicos. Contextualización, actualidad y futuro, Granada, Comares.

Вот, Н. (2005): "Dialogue interpreting as a specific case of reported speech", Interpreting, 7 (2), págs. 237-262.

Clavero Curado, C. (2011): "La documentación sanitaria para personas no hispanohablantes en Andalucía. Análisis y propuestas", en C. Valero Garcés et al. (eds.), Traducción e Interpretación en los Servicios Públicos en un mundo INTERcoNEcTado. TISP EN INTERNET/Public Service Interpreting and Translation in the Wild Wired World-PSIT in WWW, Alcalá de Henares, Universidad de Alcalá, accesible en: <http://www3.uah.es/master-tisp-uah/publicaciones-tisp-grupofitispos/>.

DAlila, N. (2008): "Un estudio de campo realizado en tres hospitales de la provincia de Alicante: impresiones sobre el papel del intérprete”, en C. Valero Garcés (ed.), Investigación y Práctica en Traducción e Interpretación en los Servicios Públicos. Desafíos y Alianzas, Alcalá de Henares, Universidad de Alcalá, accesible en: <http://www3.uah.es/master-tisp-uah/publicaciones-tisp-grupofitispos/>.

DiCKINSON, J. C. (2010): Interpreting in a community of practice: A sociolinguistic study of the signed language interpreter's role in workplace discourse, Edinburgh, HeriotWatt University.

Duranti, A. y Goodwin, Ch. (eds.) (1992): Rethinking Context. Language as an Interactive Phenomenon, Cambridge, Cambridge University Press.

Faya Ornia, G. (2011): "Trabajo de campo: tecnologías empleadas en los servicios de interpretación de los hospitales (Londres, Düsseldorf y Madrid) y experiencias de los usuarios", en C. Valero Garcés et al. (eds.), Traducción e Interpretación en los Servicios Públicos en un mundo INTERcoNEcTado. TISP EN INTERNET/Public Service Interpreting and Translation in the Wild Wired World-PSIT in WWW, Alcalá de Henares, Universidad de Alcalá, accesible en <http:// www3.uah.es/master-tisp-uah/publicaciones-tisp-grupo-fitispos/>.

Giddens, A., Duneier, M. y Appelbaum, R. (2009): An introduction to sociology, London, W. W. Norton \& Company.

Goldfarb, M. (2005): Ahmad's Peace, Ahmad's War: Surviving under Sadaan. Dying in 'the New Iraq', New York: Carroll and Carroll. 
Hale, S. (2005): “The interpeter's identity crisis”, en J. House, M. R. Ruano y N. Baumgarten (eds.), Translation and the Construction of Identity, Seoul, IATIS Yearbook, págs. 14-29.

Hale, S. (2007): Community Interpreting, Basingstoke, Palgrave McMillan.

Ilie, L., Jaime PÉRez, A. y Vitalaru, B. (2014): “Actuación del intérprete-mediador en el ámbito sanitario en situaciones comunicativas problemáticas y conflictos culturales: análisis y comparación entre intérpretes con y sin formación en diferentes pares de lenguas (español-árabe, francés, inglés y rumano)”, Panace@, $5(1)$.

ILLIE, L. (2014): "Mediación e interpretación de inglés y rumano en el proyecto InterMed", en C. Valero Garcés, (Re)considerando ética e ideología en situaciones de conflicto/(Re)visiting ethics and ideology in situations of conflict, Alcalá de Henares, Universidad de Alcalá, accesible en: <http://www3.uah.es/master-tispuah/publicaciones-tisp-grupo-fitispos/>.

Inghilleri, M. (2011): Interpreting Justice: Ethics, Politics and Language, Abingdon, Routledge.

INGHILLERI, M. (2005): "Mediating zones of uncertainty: Interpreter agency, the interpreting habitus and political asylum adjudication”, The Translator, 11 (1), págs. 69-85.

IsASA, L. (2014): "Formación del profesional sanitario en la comunicación con el paciente extranjero: estrategias y recursos", Panace@, 5(1).

Jaime, A. (2014): "Acogida del Servicio de Interpretación en Centros de Salud de Parla: primeras impresiones”, en C. Valero Garcés (ed.), (Re)considerando ética e ideología en situaciones de conflicto/(Re)visiting ethics and ideology in situations of conflict, Alcalá de Henares, Universidad de Alcalá, accesible en: <http://www3. uah.es/master-tisp-uah/publicaciones-tisp-grupo-fitispos/>.

JimÉnez Hortelano, L. (2014): "Mediación e Interpretación de Chino en el proyecto INTERMED”, en C. Valero Garcés (ed.), (Re)considerando ética e ideología en situaciones de conflicto/(Re)visiting ethics and ideology in situations of conflict, Alcalá de Henares, Universidad de Alcalá, accesible en: <http://www3.uah.es/ master-tisp-uah/publicaciones-tisp-grupo-fitispos/>.

Jiménez, S. y Murgu, D. (2016): "The use of simulation exercises based on recordings for telephone and onsite interpreter translator", en C. Valero Garcés et al. (eds.), Public Service Interpreting and Translation (PSIT): Training, Testing and Accreditation/Traducción e Interpretación en los Servicios Públicos (TISP): Formación, evaluación y acreditación / (usb format), Alcalá de Henares, Universidad de Alcalá.

KEARnS, J. (2006). "Mohammed Yousry: Translation and academic freedom under attack", IATIS Bulletin, 3, págs. 2-3.

KENINGSON-Kristy, J. (2006): "Letter to the editor: More details on the Mohammed Yousry case”, ATA Chronicle (February), págs. 10-11.

LAFrANCHI, H. (2006): "Remembering Alan: A tribute to Jill Carroll's interpreter", The Christian Science Monitor, 6 March: 1, pág. 12.

LÁZARo GutiÉrRez, R. (2010): Análisis del discurso institucional entre hablante nativo (HN) y hablante no nativo (HNN) en los servicios públicos, Alcalá de Henares, Universidad de Alcalá. Tesis Doctoral. 
MARnPae, M. (2014): "Materiales multilingües en el ámbito sanitario en España", Panace@, 5(1).

Nevado Llopis, A. (2014): "Barreras lingüísticas y culturales que dificultan la comunicación intercultural en el ámbito de la salud reproductiva”, Panace@, $5(1)$.

Nevado Llopis, A. (2016): "Acquisition and Development of Medical Interpreters' Main Competences. A Necessary Step for Professionalization”, en C. Valero Garcés et al. (eds.), Public Service Interpreting and Translation (PSIT): Training, Testing and Accreditation/Traducción e Interpretación en los Servicios Públicos (TISP): Formación, evaluación y acreditación / (usb format), Alcalá de Henares, Universidad de Alcalá.

PenA, C. (2005): "Communication between foreign doctors and patients", en C. Valero Garcés (ed.), Traducción como mediación entre lenguas y culturas/Translation as mediation or how to bridge linguistic and cultural gaps, Alcalá de Henares Universidad de Alcalá, accesible en: <http://www3.uah.es/master-tisp-uah/ publicaciones-tisp-grupo-fitispos/>.

Pena, C. y Porto, L. (2008): "Interpreting doctors and patients' mental images of illness in community healthcare", en C. Valero Garcés (ed.), Investigación y Práctica en Traducción e Interpretación en los Servicios Públicos. Desafíos y Alianzas, Alcalá de Henares, Universidad de Alcalá, accesible en: <http://www3.uah.es/ master-tisp-uah/publicaciones-tisp-grupo-fitispos/>.

PÉRez Estevan, E. (2016): "Balance of power between the interpreter and the healthcare professional? Steps for the necessary interpreter's accreditation”, en C. Valero Garcés et al. (eds.), Public Service Interpreting and Translation (PSIT): Training, Testing and Accreditation/Traducción e Interpretación en los Servicios Públicos (TISP): Formación, evaluación y acreditación / (usb format), Alcalá de Henares, Universidad de Alcalá.

Prieto, A. (2008): "La interpretación telefónica en los servicios sanitarios públicos. Estudio del caso: el servicio de 'conversación a tres' del Hospital Carlos Haya de Málaga”, en C. Valero Garcés (ed.), Investigación y Práctica en Traducción e Interpretación en los Servicios Públicos. Desafios y Alianzas, Alcalá de Henares, Universidad de Alcalá, accesible en: <http://www3.uah.es/master-tispuah/publicaciones-tisp-grupo-fitispos/>.

RAGA, F. (2014): "Etnopsiquiatría como mediación intercultural”, Panace@, 5(1).

RAGA, F. (2005): "Médicos, marabouts y mediadores: problemas de comunicación intercultural en la atención sanitaria a los inmigrantes de origen senegalés", en C. Valero Garcés (ed.), Traducción como mediación entre lenguas y culturas/ Translation as mediation or how to bridge linguistic and cultural gaps, Alcalá de Henares, Universidad de Alcalá, accesible en: <http://www3.uah.es/master-tispuah/publicaciones-tisp-grupo-fitispos/>.

Rodríguez NavazA, B. (2008): África, sangre y VIH: el papel del mediador en la resolución de conflictos culturales específicos en el ámbito sanitario", en C. Valero Garcés (ed.), Investigación y Práctica en Traducción e Interpretación en los Servicios Públicos. Desafios y Alianzas, Alcalá de Henares, Universidad de Alcalá, accesible en: <http://www3.uah.es/master-tisp-uah/publicaciones-tisp-grupofitispos/>. 
SAAR, E. y NovaK, V. (2005): Inside the Wire: A Military Intelligence Soldire's Eyewitness Account of Life at Guantánamo, New York, Penguin.

SÁnCHEZ-PEdREÑo, A. (2014): "La comunicación interlingüística y la interpretación en el Hospital Clínico-Universitario Virgen de la Arrixaca (HCUVA). Investigación para la acción”, Panace@, 5(1).

SÁnchez-Reyes, S., Durán Martínez, R. y Martín Casado, M. (2002): "Argumentos a favor de la implantación de un servicio de intérpretes en los hospitales”, en C. Valero Garcés y G. Mancho (eds.), Traducción e Interpretación en los Servicios Públicos: Nuevas necesidades para nuevas realidades. Community Interpreting and Translating; New Needs for New Realities, Alcalá de Henares, Universidad de Alcalá, accesible en: <http://www3.uah.es/master-tisp-uah/publicacionestisp-grupo-fitispos/>.

Sánchez-Reyes, S. y Martín CaSAdo, M. (2005): "Intérpretes profesionales e intérpretes 'ad hoc' en un entorno sanitario”, en C. Valero Garcés (ed.), Traducción como mediación entre lenguas y culturas/Translation as mediation or how to bridge linguistic and cultural gaps, Alcalá de Henares, Universidad de Alcalá, $<$ http://www3.uah.es/master-tisp-uah/publicaciones-tisp-grupo-fitispos/>.

STAHUJAK, Z. (2000): "Violent distortions: bearing witness to the task of the wartime translators", TTR: Traduction, Terminology, Rédaction, 13 (1), págs. 37-51.

TAIBI, M. y MARTIN, A. (2012): "Court Translation and Interpreting in Times of 'the War on Terror': The case of Taysir Alony", Translation and Interpreting, $4(1)$.

VALEnCIANo-Rodríguez, U. y Rodríguez-BARRios, F. (2011): "Las TICSs como recurso en la interpretación de lengua de signos en la Comunidad de Madrid”, en C. Valero Garcés et al. (eds.), Traducción e Interpretación en los Servicios Públicos en un mundo INTERcoNEcTado. TISP EN INTERNET/Public Service Interpreting and Translation in the Wild Wired World-PSIT in WWW, Alcalá de Henares, Universidad de Alcalá, accesible en: <http://www3.uah.es/master-tisp-uah/ publicaciones-tisp-grupo-fitispos/>.

VALERo Garcés, C. (1997): “¿Traducción e interpretación en servicios públicos? ¿De qué me hablas? ¿Una nueva especialización?”, en C. Valero Garcés et al. (eds.), Nuevas Tendencias y Aplicaciones de la Traducción, Alcalá de Henares, Universidad de Alcalá, págs. 267-277.

Valero Garcés, C. (2002): "Interaction and Conversational Constrictions in the Relationships between Suppliers of Services and Immigrant Users". Pragmatics 12 (4), págs. 469-496.

VAlero Garcés, C. (2004): "Procesos de acomodación lingüística e intercambio de roles en conversaciones institucionales. El caso de las consultas entre personal sanitario y pacientes hablantes no nativos de español (PHNN)", en J. Varela et al. (eds.), Lengua y Sociedad: Lingüistica Aplicada en la era global y multicultural, Santiago de Compostela, Universidad de Santiago de Compostela, págs. 522-531.

VAlero Garcés, C. (2005): "Comunicación Interlingüistica en los otros Servicios Públicos en España. Hospitales y centros de salud en la zona centro. Realidad y futuro", en C. Valero Garcés (ed.), Traducción e interpretación en los servicios públicos. Contextualización, actualidad y futuro, Granada, Comares. 
VAlero Garcés, C. (2005): "Doctor-Patient Consultations in Dyadic and Triadic Exchanges”, Interpreting, 7 (2), págs. 193-210.

VAlero Garcés, C. (ed.) (2005): Traducción e interpretación en los servicios públicos. Contextualización, actualidad y futuro, Granada, Comares.

VAlero GarcÉs, C. (ed.). (2005): Traducción como mediación entre lenguas y culturas/Translation as mediation or how to bridge linguistic and cultural gaps, Alcalá de Henares, Universidad de Alcalá.

VALERo GARCÉs, C. (2008): "Hospital interpreting practice in the classroom and the workplace", en C. Valero Garcés y A. Martin (eds.), Crossing Borders in Community Interpreting. Definitions and dilemmas, Amsterdam/Philadephia, John Benjamins, págs. 165-185.

VAlero Garcés, C. (2008): "Salud, Comunicación e Inmigración”, en A. M. Bañón y J. Fornieles (eds.), Manual sobre Comunicación e Inmigración, San Sebastián, Gakoa, págs. 381-402.

VAlero Garcés, C. (2010): "La conversación de contacto en contextos institucionales: la consulta médica”, Oralia, 11, págs. 107-131.

VAlero Garcés, C. (2013): "Comunicación con población extranjera en el ámbito sanitario. Iniciación a una base de datos sobre artículos de investigación”, Panace@, 14 (37).

VAlero GARCÉs, C. (2015): "Health communication and multicultural communities. Topics on intercultural communication for healthcare professionals", Cambridge, Cambridge University Press.

Valero Garcés, C. y Mancho, G. (eds.) (2002): Traducción e Interpretación en los Servicios Públicos: Nuevas necesidades para nuevas realidades. Community Interpreting and Translating; New Needs for New Realities, Alcalá de Henares, Universidad de Alcalá.

VALERo GARCÉs, C. y TAIBI, M. (2004): “Análisis de la interacción comunicativa en contextos institucionales entre proveedores de servicios, usuarios e intermediarios lingüísticos", Oralia, 7, págs. 207-228.

Valero Garcés, C. y Downing, B. (2007): "Modes of communication between suppliers of services and non-native English-speaking users: doctor-patient interaction", en G. Garzone y C. Ilie (eds.), The Use of English in Institutional and Business Settings, Berna, Peter Lang.

VALERO GARCÉS y LÁZARO, R. (2008): "Investigación sobre la calidad de la comunicación en la atención sanitaria al inmigrante", en C. Valero Garcés (ed.), Investigación y Práctica en Traducción e Interpretación en los Servicios Públicos. Desafíos y Alianzas. Alcalá de Henares, Servicio de Publicaciones de la Universidad de Alcalá, accesible en: <http://www3.uah.es/master-tisp-uah/publicaciones-tispgrupo-fitispos/>.

VAlero Garcés, C. y ABKARI, A. (2010): "Learning from practice. Interpreting at the $11 \mathrm{M}$ terrorist attack trial”, Translation and Interpreting, 2 (2).

VAlero GarcÉs, C. y WAHL, E. (2014): "La voz de los profesionales de la salud y de los usuarios extranjeros sobre choques culturales”, Panace@, 5(1).

Valero Garcés, C., Lázaro Gutiérrez, R. y Del Pozo Triviño, M. (2015): "Interpretar en casos de violencia de género en el ámbito médico", en M. Del Pozo 
Triviño y C. Toledano (eds.), Interpretación en contextos de violencia de género, Valencia, Tirant lo Blanch, págs. 192-238.

Valero Garcés, C. y Tipton, R. (eds.) (2017): Ideology, Ethics and Policy Development in Public Service Interpreting and Translation, Bristol, UK, Multilingual Matters.

VARGAS URPI, M. (2011): "The Interdisciplinary Approach in Community interpreting research", New Voices in Translation Studies, 7, págs. 47-64.

Vitalaru, B. (2011): "Herramientas wiki, trabajo colaborativo y elaboración de materiales multilingües de carácter sanitario en un máster universitario”, en C. Valero Garcés et al. (eds.), Traducción e Interpretación en los Servicios Públicos en un mundo INTERcoNEcTado. TISP EN INTERNET/Public Service Interpreting and Translation in the Wild Wired World-PSIT in WWW, Alcalá de Henares, Universidad de Alcalá, accesible en: <http://www3.uah.es/master-tisp-uah/publica ciones-tisp-grupo-fitispos/>. 